\title{
Operant Conditioning in Lymnaea: Evidence for Intermediate- and Long-term Memory
}

\author{
Ken Lukowiak, Nimet Adatia, Darin Krygier, and Naweed Syed ${ }^{\mathbf{1}}$ \\ Neuroscience and Respiratory Research Groups, Faculty of Medicine, University of Calgary, Calgary, Alberta T2N 4N1 Canada
}

\begin{abstract}
Aerial respiration of the pond snail, Lymnaea stagnalis, can be operantly conditioned; however, the parameters necessary to produce long-term (LTM) or intermediate term memory (ITM) have not previously been investigated. We conducted training using procedures that varied in the duration of the training session, the number of training sessions per day or the amount of time between subsequent training sessions (SI). We found that by varying the duration and frequency of the training session learning could be differentially produced. Furthermore, the ability to form LTM was dependent not only on the duration of the training session was also the interval between training sessions, the SI. Thus it was possible to produce ITM, which persists for up to $3 \mathrm{hr}$, and not form LTM, which persists at least $18 \mathrm{hr}$. Learning, ITM, and LTM can be differentially produced by altering the SI, the duration of the training session, or the number of training sessions per day. These findings may allow us to begin to elucidate the underlying neural mechanisms of learning, ITM, and LTM.
\end{abstract}

Operant or instrumental conditioning is a form of associative learning produced by the presentation of a reinforcing stimulus to the subject after the occurrence of a particular behavior (Mackintosh 1974). Thus the animal learns to get a reward by operating on its environment. Reinforcement can be negative, leading to a decrease in the appearance of the behavior or positive, resulting in an increase in the animal's tendency to perform the behavior (Dickinson 1980). Operant conditioning has not been studied to the same extent as classical conditioning or nonassociative behaviors in molluscan model systems (Mpitsos and Lukowiak 1985; Nargeot et al. 1999). Thus our understanding of the various parameters affecting learning and memory of this form of associative learning, especially at the neuronal level, are not as well understood as those of nonassociative or classical conditioning.

Learning and memory are not a unitary process; rather, they are a constellation of distinct and independent processes (Milner et al. 1998). Learning is defined here as an experience-derived adaptive change in a behavioral program, whereas memory is defined as the ability to store and recall previous experiences. In the taxonomy of memory there are three species: short-term memory (STM, lasting minutes), intermediate-term memory (ITM, 2-3 hr), and long-term memory (LTM, >18 hr) (Rosenzweig et al. 1993). Which form of memory is evoked depends on the nature, intensity, and pattern of training (Lechner et al. 1999). The process by which the transient memory, STM is converted to a more stable, longer lasting memory, LTM, is called consolidation and is dependent on altered gene activity and

${ }^{1}$ Corresponding author.

E-MAIL lukowiak@ucalgary.ca; FAX 403-283-2700. new protein synthesis (Abel et al. 1998; Carew 1996; Martin et al. 1997: Mauelshagen et al. 1998; Tully 1998).

For over 100 years it has been known that altering the interval between training sessions can interfere with the consolidation process (Lechner et al. 1999). Thus, after learning occurs, memory remains vulnerable to disruption until a certain length of time has elapsed. This process has given rise to the concept of massed versus spaced training and the effect on memory retention. While both training procedures can lead to the same level of learning, memory persists longer with spaced training than it does with massed training (e.g., cramming for an exam). Thus, for example, in studies of agoraphobia in humans, it has been shown that massed training results in greater reductions in anxiety than spaced training, but that the latter results in better long-term retention (Rowe and Craske 1998). Similar data have been obtained in Aplysia habituation and sensitization studies (Carew et al. 1972) as well as in studies of other vertebrate and invertebrate species (Hermitte 1999; Lechner et al. 1999).

We looked at the effects of altering the session interval (SI) between training sessions on the establishment of LTM in our Lymnaea model system. Aerial respiratory behavior and its ability to be operantly conditioned provide a useful model system for the study of the neuronal basis of learning and memory. There are a number of reasons for this usefulness. The first reason is that we are conditioning a rhythmic, homeostatic behavior, aerial respiration. Lymnaea respire bimodally, using both cutaneous and aerial respiration, thus we can temporarily inhibit or suppress aerial respiration without endangering the viability of the snail. The second reason is that a three-cell network of identified interneurons mediates the behavior (Syed et al. 1990). Moreover, the

LEARNING \& MEMORY 7:140-150 @ 2000 by Cold Spring Harbor Laboratory Press ISSN1072-0502/00 \$5.00

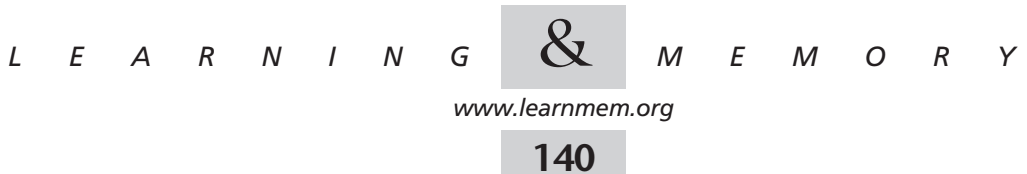


sufficiency and necessity of these neurons for the mediation of this behavior have been established (Syed et al. 1992). Neural correlates of operant conditioning and its LTM have already been found in both isolated ganglia preparations obtained from trained animals (Spencer et al. 1999a) and semi-isolated preparations where the behavior and accompanying neural activity can be recorded from simultaneously (Lukowiak et al. 1999).

Although we have been able to demonstrate operant conditioning of this behavior we do not know the constraints on the induction and maintenance of learning and the different forms of memory. By that we mean that, we are uncertain how the parameters of training session duration (and thus the number of reinforcing stimuli delivered to the animals), frequency of training, and interval between training sessions interact to allow the establishment of learning and memory. We are also uncertain as to whether this model system exhibits ITM. If we are to use this model system to explore the neuronal mechanisms of learning and memory, then we need to understand better the process of learning and the various forms of memory possessed by the system.

Therefore, the goal of this study was to investigate the effects of training session duration (and thus the number of reinforcing stimuli), frequency of training, and length of interval between training sessions on the formation and persistence of ITM and LTM. We operationally defined LTM and ITM on the basis of whether the number of attempted pneumostome openings in the session following an 18-hr (for LTM) or 2- to-4-hr interval (for ITM) were not significantly different from each other, but were different from the response in session 1 .

\section{RESULTS}

\section{The Effect of the Duration of Training Session on Learning and Memory}

The first aim of this study was to determine the effect of the duration of the training session on the acquisition and retention of the learned behavior. To accomplish this task, we first used the procedure 1 protocol. Thus, three separate groups of snails underwent one training session per day for 5 days (Fig. 1 ). A training session of $0.25 \mathrm{hr}(n=10)$ resulted in neither learning nor LTM. The second cohort of animals $(n=18)$ was trained with a training session duration of $0.5 \mathrm{hr}$ and this session, too, resulted in neither learning nor LTM. However, the third cohort of animals ( $n=17)$, which received a 1-hr training session, exhibited both learning and LTM [ANOVA $F(4,5)=17.6837, \mathrm{P}<0.001]$. Paired comparisons showed that the number of pneumostome openings on day 2 was statistically different from the number that occurred on day 1 , indicating that learning had

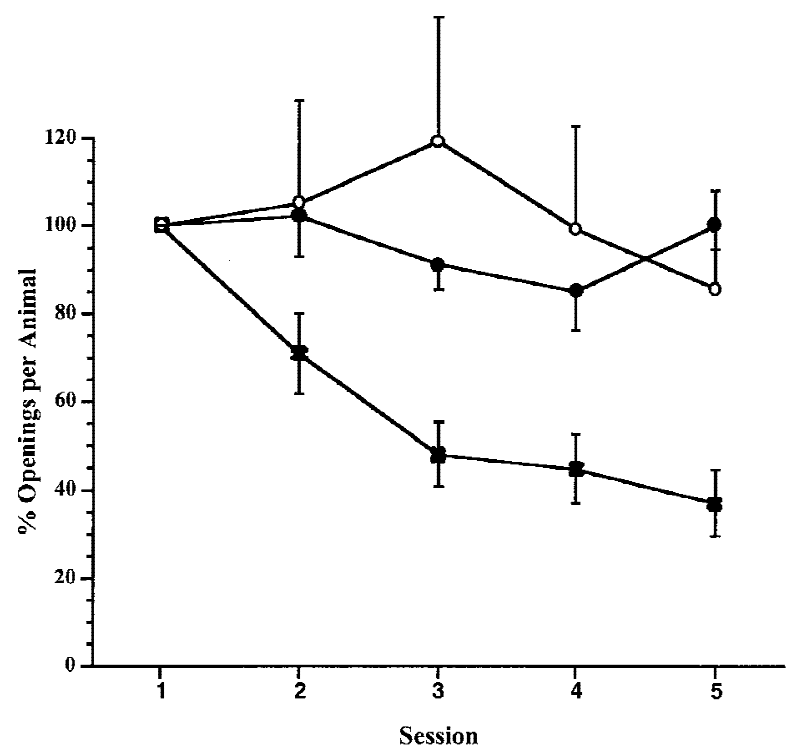

Figure 1 Learning and LTM receiving a single training session a day for 5 days. The normalized data are from three cohorts fo animals trained once a day for five days. The cohorts having a training session of 0.25 and $0.5 \mathrm{hr}$ ( $\bigcirc$ and $\bigcirc$ respectively) did not exhibit either learning or LTM, whereas the cohort receiving the 1 $\mathrm{hr}$ training session $(x)$ showed both learning and LTM.

occurred and that there was significant retention of memory $>18 \mathrm{~h}$ after session $1 ; t=3.3684, \mathrm{P}<0.01$ ).

From these data we conclude that the amount of training given per day had a significant effect on the animal's ability to acquire and retain new information. A 1-hr training session once a day is sufficient to both produce learning (as evidenced by the significant difference between the number of pneumostome openings on day 2 compared with day 1, etc.) as well as to allow the consolidation process to occur leading to the formation of LTM. Training periods of 0.5 or $0.25 \mathrm{hr}$ given once per day were insufficient to produce either learning or memory.

As was expected, the actual number of breathing attempts made by the naive snails in training session 1 was dependent on the duration of the training session. Thus the animals in the 1 -hr training session $(n=17)$ made $17.1 \pm 1.8$ S.E.M. breathing attempts, while those in the 0.5 - and $0.25-\mathrm{hr}$ sessions made $8.9 \pm 1.9$ and $3.6 \pm 0.5$, respectively.

\section{Number of Training Sessions}

Because the data obtained from animals treated with the procedure 1 protocol showed us that a training session of 1 hr produced learning and memory; we hypothesized that 1 $\mathrm{hr}$ of training split into two 0.5 sessions per day was sufficient to produce learning and memory. Therefore, we tested a cohort of animals $(n=35)$, using the procedure 2 protocol. As can be seen in Figure 2, these animals exhibited both learning and LTM [ANOVA $F(3,34)=5$. 5452, $P<0.001$; paired comparisons showed that the number of

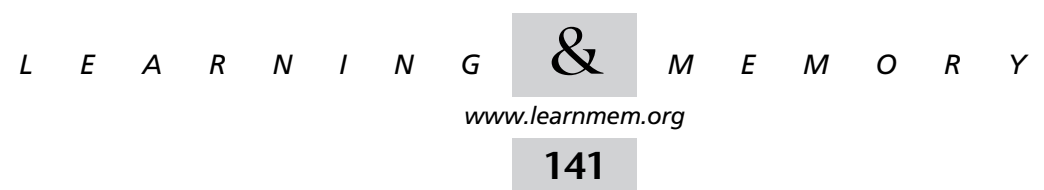




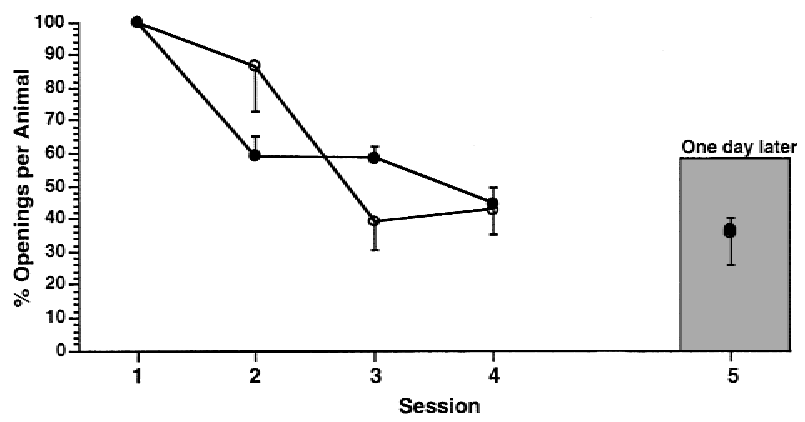

Figure 2 Learning and LTM in animals receiving two training sessions a day with an SI of $1 \mathrm{hr}$. The cohort of animals receiving the 0.25 -hr training session $(\bigcirc)$ and the cohort receiving the $0.5-\mathrm{hr}$ training session $(\mathbf{)}$ both exhibited learning and LTM.

pneumostome openings in session 4 was significantly different from in session $1(t=3.86, P<0.01)$ and that the number of openings in session 4 was not significantly different from session 5 (18 hr later; $t=-1.25, P>0.05$ ) whereas the number of openings in session 5 was significantly different from session $1 ;(t=2.86, P<0.01]$. Using the same experimental protocol (procedure 2), we determined that a $0.25-\mathrm{hr}$ duration training session twice a day for 2 days was also sufficient to produce learning and LTM. We found that even though the total time of training was reduced by half, these animals $(n=32)$ exhibited both learning and LTM [Fig. 2, ANOVA $F(3,31)=5.3245$, $P<0.001$; paired comparisons showed that the number of pneumostome openings in session 4 was significantly different from in session $1(t=3.66, P<0.01)$. Likewise, the number of pneumostome openings in session 5 (18 hr after session 4) was not significantly different from the number of openings in session $4(t=1.19, P>0.05)$ but was significantly different from session $1(t=2.39, P<0.01]$. Again, animals receiving the $0.5-\mathrm{hr}$ training session attempted to breathe more often than those receiving the 0.25 -hr training session $(11.1 \pm 2.1$ vs. $3.2 \pm 0.6$ in session 1$)$.

Then we determined whether learning and LTM could be elicited with a single day of training. To test this question we used the procedure 3 protocol. The first cohort of animals $(n=6)$ received a $1-\mathrm{hr}$ training session while the second and third cohorts received a 0.5 or $0.25-\mathrm{hr}$ training session ( $n=12$ and 14, respectively) with an SI of $1 \mathrm{hr}$. Each cohort demonstrated learning and LTM [Fig. 3. for the 1-hr group, ANOVA $F(3,11)=5.3402, P<0.01$; paired comparisons show that session 3 was significantly different from session $1(t=3.5276, P<0.01)$ but was not different from session $4(t<=0.4342, P>0.05)$ whereas session 4 was significantly different from session $1(t=3.9618, P<0.01)$. For the 0.5 -hr group ANOVA $F(3,11)=5.4558, P<0.003$; paired comparisons show that session 3 was significantly different from session $1(t=3.7836, P<0.01)$ but was not different from session $4(t=-0.70, P>0.05)$ whereas session 4 was significantly different from session 1 ( $t=3.083$,

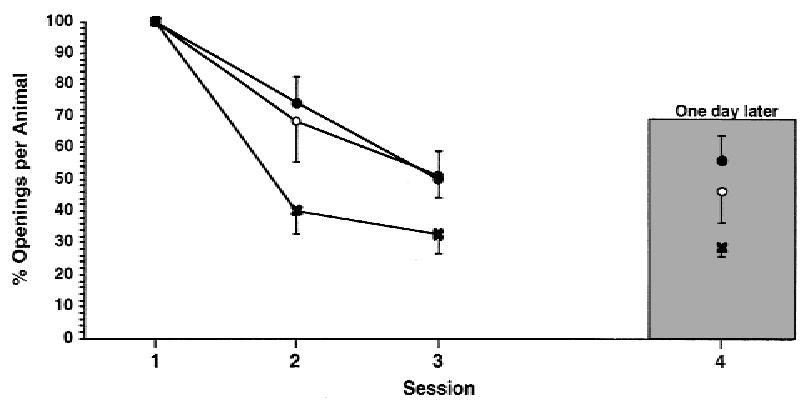

Figure 3 Learning and LTM in cohorts of animals receiving three training sessions a day with an $\mathrm{SI}$ of $1 \mathrm{hr}$. Separate cohorts of animals receiving three $0.25-\mathrm{hr}(\bigcirc)$, $0.5-\mathrm{hr}(\mathbf{O})$, or 1-hr $(\mathrm{x})$ training sessions a day, respectively, with an SI of $1 \mathrm{hr}$ exhibit both learning and LTM.

$P<0.01$, for the 0.25 -hr group ANOVA $F(3,13)=4.5911$, $P<0.007$; paired comparisons show that session 3 was significantly different from session $1(t=2.9678, P<0.01)$ but was not different from session $4(t=0.4397, P>0.05)$ whereas session 4 was significantly different from session 1 $(t=3.4074, P<0.01]$. Thus learning and LTM could be produced with only a single day of training. Again, it needs to be emphasized that animals receiving a 1-hr training session received more pokes than those receiving either a 0.5 - or $0.25-\mathrm{hr}$ training session $(16.5 \pm 2.3 ; 9 \pm 1.9$; and $4.1 \pm 0.5$, respectively).

The conclusion we reached was that animals need only to receive a total of 0.75 - $\mathrm{hr}$ of training divided into three sessions on 1 day with an SI of 1 -hr to learn and exhibit LTM. Moreover, even when animals only received a relatively few reinforcing stimuli (i.e., the 0.25 -hr group) they were still able to learn and remember.

\section{Elapsed Time Between Sessions (SI)}

Next, we examined the effects of altering the SI on the ability of the animals to learn and remember while keeping the duration of the training session constant. First we determined what the effect of decreasing the SI from 1 to 0.5 -hr would be using the $0.25-\mathrm{hr}$ duration training session three sessions per day. A naïve cohort of animals $(n=14)$ received three 0.25 -hr duration training sessions with an SI of $1 \mathrm{hr}$. These animals showed significant learning and LTM when tested 1 day later [Fig. 4 ANOVA $F(3,13)=4.5911$, $P<0.007$; paired comparisons showed that session 4 was significantly different from session $1(t=3.4074, P<0.01)$ but was not significantly different from session $3(t=0.4397$, $P>0.05)]$. As expected, in these same animals, session 3 was significantly different from session 1 showing that learning occurred $(t=5.9891, P<0.01)$. However, when another cohort of animals $(n=22)$ received three 0.25 -hr duration training sessions with an SI of $0.5 \mathrm{hr}$, learning, but not LTM, resulted [Fig. 5, ANOVA $F(2,21)=20.6237, P<0.0001$; session 3 was significantly different from session $1(t=6.1469$,

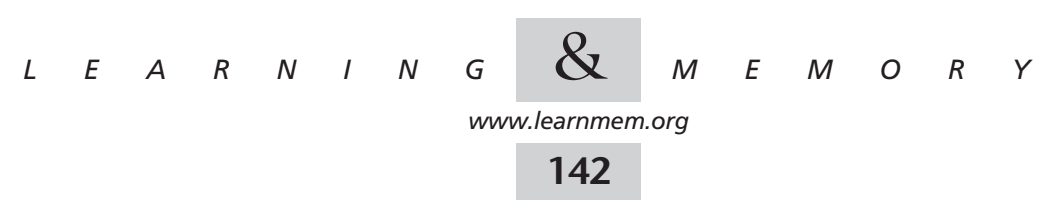




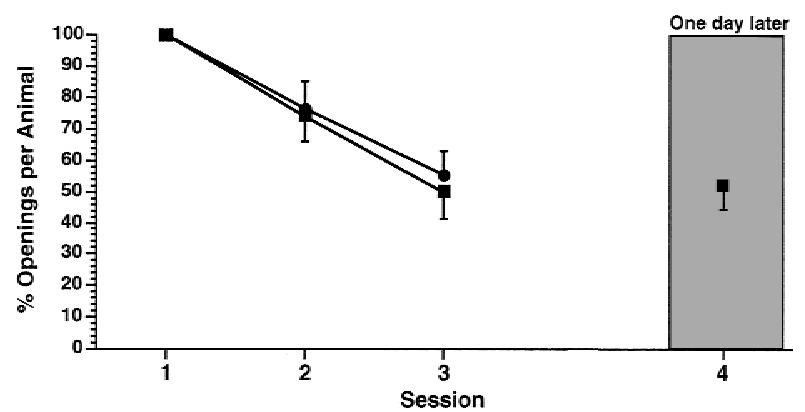

Figure 4 The affect of altering the $\mathrm{SI}$ from $1 \mathrm{hr}$ to $0.5 \mathrm{hr}$ on the establishment of LTM. Two cohorts of animals each received three 0.25-hr training sessions a day. Only the cohort whose training procedure included the 1-hr SI showed LTM (ם). The cohort whose training procedure used an $\mathrm{SI}$ of $0.5 \mathrm{hr}$ shwoed learning but not $\operatorname{LTM}(\mathbf{\bullet})$.

$P<0.01$. However, there was no significant difference between session 4 and session $1(t=0.5943, P>0.05)$, but there was a significant difference between session 3 and session $4(t=4.6118, P=0.01)]$. Thus, while learning occurs with three 0.25 -hr training sessions per day using either a 0.5 - or 1.0-hr SI, LTM is only observed with an SI of $1 \mathrm{hr}$. A 0.5 -hr SI apparently occludes the consolidation process. Remember however, that this short duration training session is not sufficient to produce learning or LTM when the SI was 1 day (Fig. 1).

Similar data were obtained with the 0.5 -hr duration training session [Fig. 5, ANOVA $F(3,21)=7.1183, P<0.002$; session 4 was not significantly different from session 1 $(t=1.6763, P>0.05)$ but was different from session 3 $(t=2.0787, P \ll 0.05)$, whereas session 3 was significantly different from session 1 ( $t=7.1482, P<0.01$ ]. That is, learning, but not LTM, occurred with an SI of $0.5 \mathrm{hr}$. However, the cohort of animals trained with a 1-hr training session produced both learning and LTM even with an SI of $0.5 \mathrm{hr}$ (Fig. 6, ANOVA $F(3,9)=3.9166, P<0.01$; session 4 was significantly different from session $1(t=3.3144, P<0.01)$ but was not

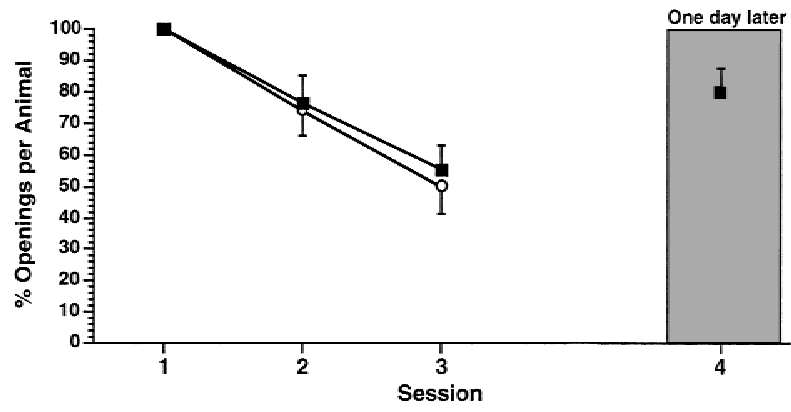

Figure $5 \mathrm{SI}$ and the establishment of LTM with a training session duration of $0.5 \mathrm{hr}$. Two cohorts of animals received three $0.5 \mathrm{hr}$ training session a day. Only the cohort that had an SI of $1.0 \mathrm{hr}$ exhibited both learning and LTM $(O)$, whereas the cohort having the $\mathrm{SI}$ of $0.5 \mathrm{hr}$ only exhibited both learning ( $\mathbf{\square})$.

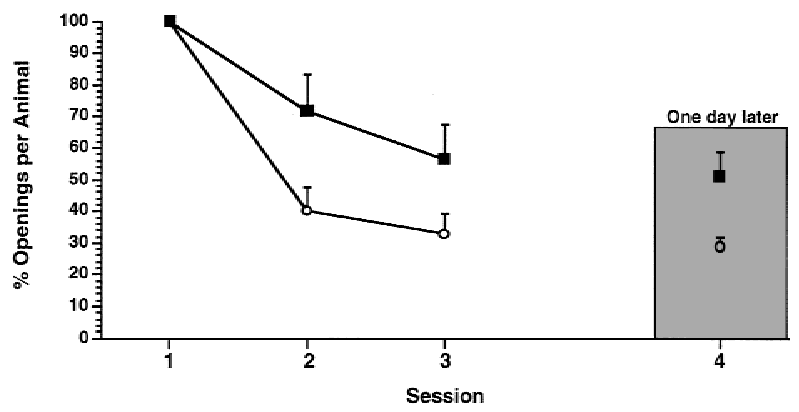

Figure $6 \mathrm{SI}$ and the establishment of LTM with a training session duration of $1 \mathrm{hr}$. Two cohorts of animals received three training sessions a day. Both learning and LTM were observed irrespective of which SI was used (O), $1 \mathrm{hr}(\mathbf{\square})$.

significantly different from session $3(t=1.0388, P<>0.05)$ whereas session 3 was significantly different from session 1 $(t=3.5641, P<0.01)$.

\section{ITM}

Decreasing the SI from $1 \mathrm{hr}$ to $0.5 \mathrm{hr}$ between the three training sessions on one day interfered with the establishment of LTM when either the 0.25- or 0.5-hr duration training session was used (Figs. 4 and 5). We hypothesized, however, that ITM (memory lasting at most $4 \mathrm{hr}$ ) would be present in these animals. Therefore, we tested for ITM 2 or $3 \mathrm{hr}$ after the third training session.

First we tested a cohort of animals $(n=20)$ with three $0.25-\mathrm{hr}$ duration training sessions per day with an SI of 0.5 hr. As can be seen (Fig. 7) when tested $2 \mathrm{hr}$ after the last training session, these animals showed both learning and significant retention of the learned behavior [i.e., memory [ANOVA $F(3,19)=22.1896, P<0.001$; session 4 was significantly different from session $1(t=4.2672, P<0,01)$ but was not significantly different from session $3(t=1.638$, $P \ll 0.05$ ), however, session 3 was significantly different from session $1(t=4.6732, P<0.01]$. A second cohort $(n=24)$ was tested in a similar manner, but in this group, we tested the retention of the learned behavior $3 \mathrm{hr}$ after the last training session. Again, these animals exhibited learning and significant retention of memory [ANOVA $F(3,23)=18.6834, P<0.001$; session 4 was significantly different from session $1(t=3.904, P<0.01)$ but was not significantly different from session $3(t=0.3877, P>0.05)$. However, session 3 was significantly different from session $1(t=4.1031, P<0.01]$. A third cohort of animals $(n=20)$ was tested by use of the same procedure, but in these animals, we tested for the retention of the learned behavior 4 $\mathrm{hr}$ after the last training session. These animals although demonstrating learning, did not exhibit ITM [ANOVA $F(3,19)=16.3266, P<0.001 ;$ session 4 was not significantly different from session $1(1.9504, P>0.05)$ but was significantly different from session $3(t=2.007, P>0.05)$ whereas session 3 was significantly different from session 1

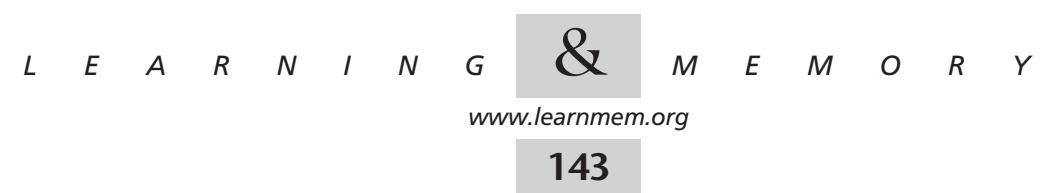




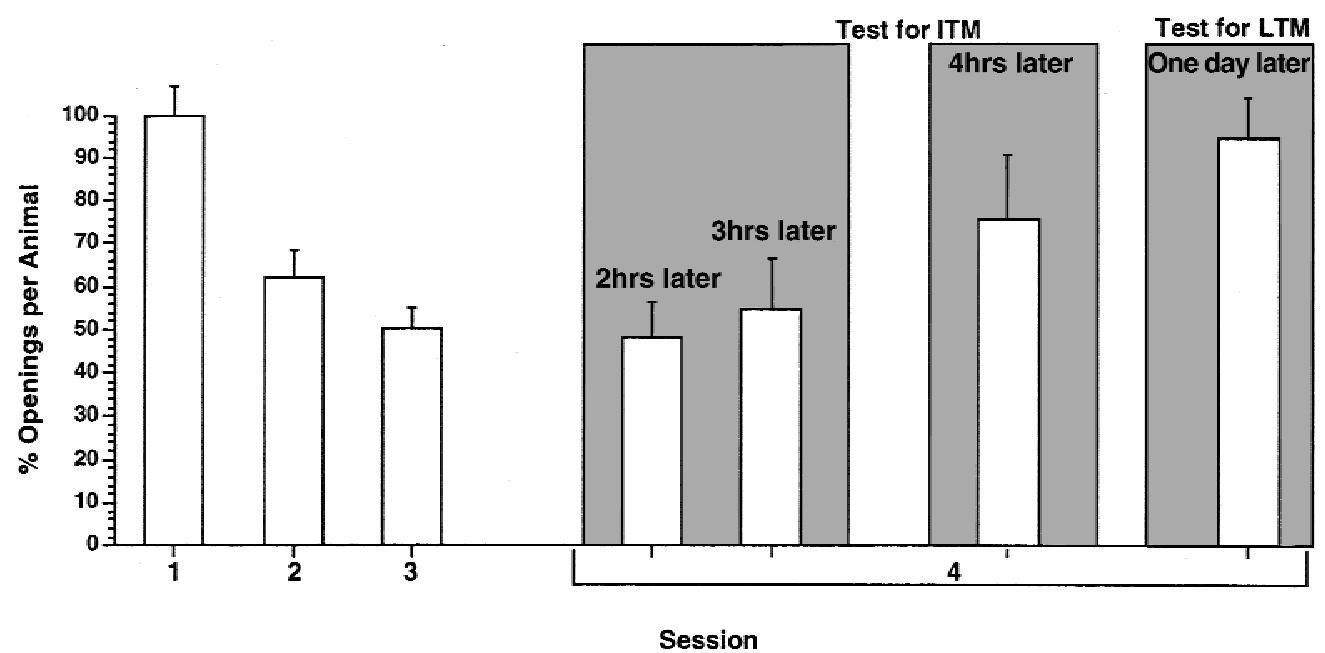

Figure 7 The persistence of ITM. Four cohorts of animals recieved three 0.25-hr training sessions a day with an SI of $0.5 \mathrm{hr}$. The cohort tested 1 day later failed to exhibit LTM. A separate cohort tested $4 \mathrm{hr}$ after the last training sessions also failed to exhibit any memory. However, the cohorts tested 2 or $3 \mathrm{hr}$ after the last training session, respectively, exhibited ITM. Thus, ITM persisted for $3 \mathrm{hr}$ under these conditions.

$(t=2.4362, P<0.01)]$. We conclude that ITM was present but that it decayed sometime between 3 and $4 \mathrm{hr}$ after the final training session. A final cohort of animals was trained, and we tested for retention of memory $18 \mathrm{hr}$ after the third training session. These data are similar to those presented in Figure 3 .

Next we determined whether increasing the duration of the training session (from 0.25 -to $0.5 \mathrm{hr}$ ) had an effect on the establishment or persistence of ITM. Significant retention of the learned behavior (i.e., ITM) was evident at both 2 [ANOVA $F(3,11)=10.5652, P<0.001$; session 4 was significantly different from session $1(t=3.0322, P<0.01)$ but was not significantly different from session $3(t=0.0551$, $P>0.05$ ] and $3 \mathrm{hr}$ later [ANOVA $F(3,11)=15.8768, P<0.001$; session 4 was significantly different from session $1(t=4.0897$, $P<0.01)$ but was not significantly different from session 3 $(t=0.9524, P>0.05)]$. However, ITM was not evident $4 \mathrm{hr}$ later [ANOVA $F(3,11)=7.1183, P<0.002$; session 4 was not significantly different from session $1(1.6763, P>0.05)$ but was significantly different from session $3(t=2.0787, P<0.05$ ] $n=12$ in all cases; Fig. 8 ). In all three cohorts, the number of attempted pneumostome openings (i.e., the number of pokes to the pneumostome) in session 3 was significantly different from session 1 , indicating that learning occurred $(P<0.01$ in all three cases). Again the data from a final cohort tested $>18$ hr later showed no LTM as in Figure 4.

Together, all these data demonstrate that learning as well as ITM and LTM can be differentially elicited in Lym-

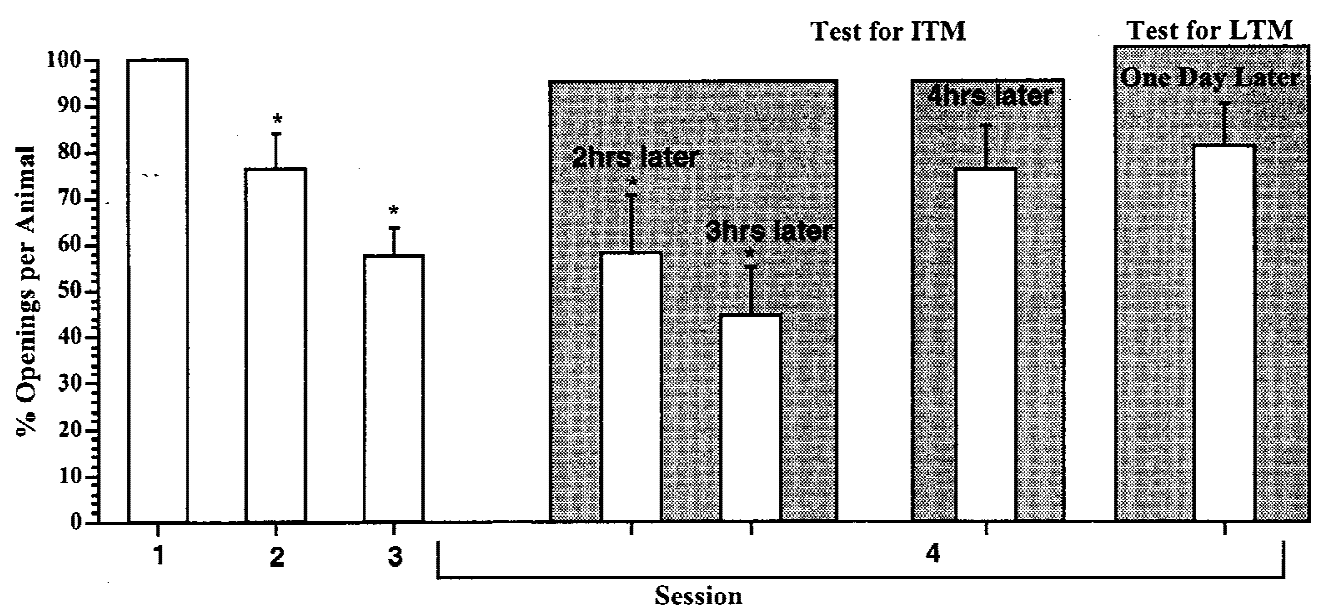

Figure 8 The persistence of ITM in snails recieving three 0.5-hr training sessions a day with an SI of 0.5-hr. Data as in Fig. 7. ITM persists up to $3 \mathrm{hr}$ after the last training session but disappears by $4 \mathrm{hr}$.

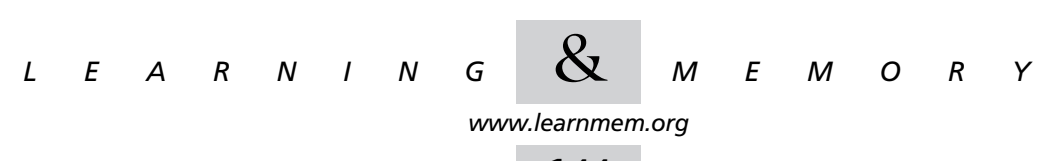


naea by altering the SI, the duration of the training session, or the number of training sessions per day.

To demonstrate that the observed changes in behavior subsequent to the various training procedures were the result of operant conditioning and thus associative learning, we ran a series of yoked controls (see Materials and Methods). Because learning occurs during the 1-hr training session, we had to use a slightly different approach to the yoked control procedure than we did for the 0.5 -and $0.25-\mathrm{hr}$ sessions. For the 1-hr training session yoked control animals $(n=10)$ we poked their pneumostome at the time the animal undergoing operant conditioning attempted to open its pneumostome (i.e., the animal to which it was yoked). Eighteen hours after the third training session, we tested the yoked control snails. In this test, we poked the yoked control animal's pneumostome when it attempted to open it. We then compared the number of attempted openings in this test session with the number of openings made by all the 1-hr snails in session 1 using procedure 3. As shown in Figure 9A ( the number of openings in the test session was almost the same as that on session 1) $t=0.3497, P>0.05)$. We used a slightly different procedure (see Materials and Methods) for the 0.5 -and 0.25 -hr session yoked control animals. Both of these groups (Fig. 9 B,C) were given a pre-test session in which we applied a poke to their pneumostome whenever they attempted to open it. Following a rest interval of $24 \mathrm{hr}$, which as we saw from the data in Figure 2 produces no learning or memory, we applied a poke to their pneumostome whenever the animal to which they were yoked attempted to open its pneumostome (procedure 3). Then we tested the yoked control animals $18 \mathrm{hr}$ later. As can be seen, neither group showed any memory; that is, the number of attempted pneumostome openings in the pre-test was not significantly different from the post-test $(0.5 \mathrm{hr}, t=0.28761, P>0.05 ; 0.25 \mathrm{hr} t=0.0273, P>0.05)$. Thus only animals that received contingent reinforcement showed learning and memory.

\section{DISCUSSION}

We demonstrated previousy that operant conditioning of aerial respiration in Lymnaea exhibits many of the characteristics of memory retention found in other preparations (Milner et al 1998; Rosenzweig et al. 1993). For example, decreasing the time interval between training sessions (SI) interferes with the consolidation process, thus blocking the establishment of LTM. In so doing, we have been able to demonstrate for the first time that another form of memory, ITM, is also present in Lymnaea. In our initial study on operant conditioning of aerial respiration in Lymnaea (Lukowiak et al. 1996) five 0.5-hr duration training sessions with an SI of at least $1 \mathrm{hr}$ were given over the course of 2.5 days. That training procedure resulted in learning and the establishment of LTM that persisted for at least 1 week. Later we extended this finding by showing that a training

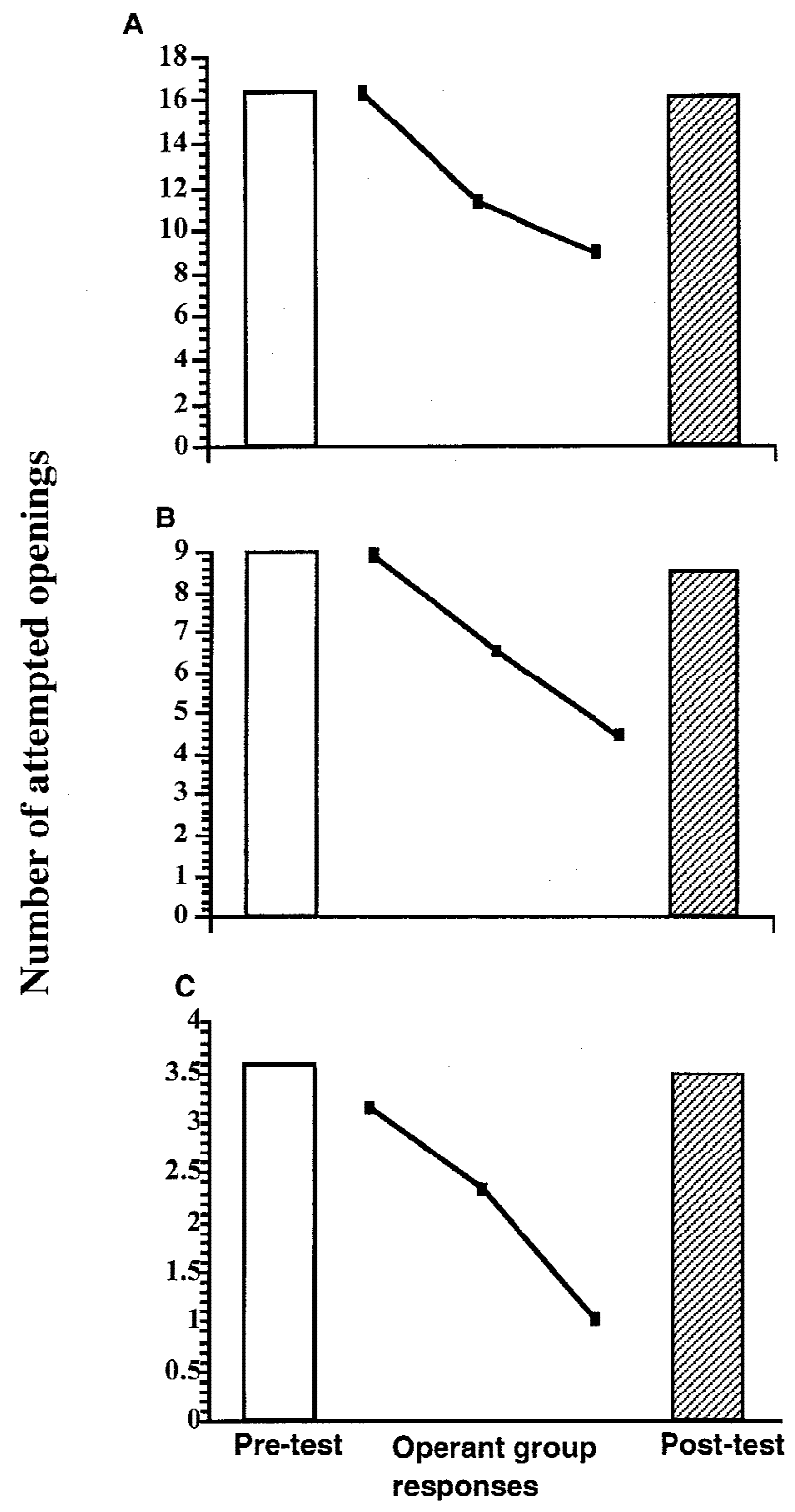

Figure 9 Yoked control animals do not exhibit learning of memory. Three cohorts of animals were given the yoked control procedure. (Open bars) Pre-test data; (hatched bar) post-test data. The data ( $)$ represent the learning curve obtained by the cohort receiving the operant training procedure (procedure 3). (A) Data from the 1-hr training session; $(B)$ 0.5- and $(C) 0.25$-hr training sessions.

procedure consisting of eight training sessions spread out over a 4-week period resulted in learning and LTM that persisted for at least 1 month (Lukowiak et al. 1998). In our previous experiments, we did not study whether decreasing the SI had any affect on the establishment of LTM or its duration and did not attempt to determine whether ITM was present.

What our initial findings did demonstrate (Lukowiak et al. 1996) was that we had a preparation that could be op-

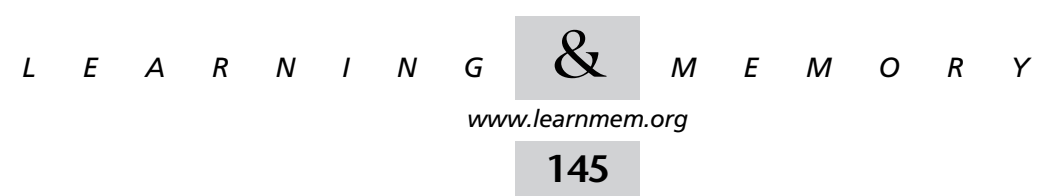


erantly conditioned. We were able to reach this conclusion by showing that yoked control preparations and hypoxic control animals did not exhibit the changes in behavior seen when there was a contingent presentation of the reinforcing stimulus with attempted pneumostome opening. The hypoxic controls (animals prevented from breathing air in the hypoxic training beaker for the entire training period) also did not suffer any observed ill effects of not being able to breath air. In fact, we have kept animals from breathing air for over $24 \mathrm{hr}$ in a hypoxic environment without any increase in observed mortality or changes in the ability of animals to perform their normal everyday functions. Thus the observed changes in behavior (the learning) and the persistence of these changes (memory) are the result of associative learning (contingent presentation of the reinforcing stimulus) and not some abnormal change in physiology or metabolism brought on by being in a hypoxic environment. It is possible, however, that, as a result of the associative learning and its memory, the animals' metabolism may undergo adaptive change. We are presently performing experiments that address this question. However, if there are such adaptive changes in metabolism, heart rate, etc. they only occur when the operant conditioning procedures are used.

The usefulness of a preparation for the study of the neural substrates of learning and memory is dependent not only on the relative ease of assessing changes in its nervous system that ultimately cause the learning and memory but also on at least two other factors. These are (1) the ease with which learning and memory are inducible and (2) the types of learning and memory that can be produced. With the results presented here we now are able to show that operant conditioning of aerial respiration in Lymnaea can be easily produced with a single day of training; and that both ITM and LTM can be differentially evoked. Thus, it is easy, quick, and straightforward to produce a form of associative learning and its long-lasting memory. Because the sufficiency and necessity of the neurons mediating the behavior that exhibits the learning and memory are already known (Spencer et al. 1999), the usefulness of this preparation has been extended.

For the memory of learned behavior to proceed from a transient, volatile STM, to a long-lasting, stable form, LTM, it must go through the consolidationprocess (Carew 1996; Tully 1998; Lechner et al.) The consolidation process may be interfered with in a number of different ways. Thus for example, the use of transcriptional or translational blockers of new protein synthesis prevents the establishment of LTM (Milner 1998; Rosenzweig 1993). However, these are rather drastic measures that an animal would not ordinarily encounter. Yet we know that not all learning results in LTM (ask any teacher). At a more basic level, we know by our own experience, that the consolidation process can be interfered with by the alteration of the interval between train- ing sessions. This information has been presented in the scientific literature for over 100 years (Lechner et al. 1999) and we suspect has been known for as long as parents have been attempting to train their offspring. That is, massed training, while resulting in a similar performance level, yields shorter lasting memories.

Does operant conditioning of aerial respiration in Lymnaea follow similar rules regarding the formation of LTM? First, we tested the effects of the duration of the training session. Few conclusions have been drawn about the session length required to form LTM. Here, we found that a single 0.25 -hr and 0.50 -hr training session once a day for 5 days was inadequate to produce learning or LTM. This inadequacy could be due to an insufficient amount of time to cause a meaningful association between the tactile stimulus to the pneumostome area and the animal's attempt to breathe. However, a training session of 1-hr duration is sufficient to both produce learning and LTM (Fig. 2). These data suggest that both the learning and the consolidation process can occur during the training period, if that period is sufficiently long.

Not surprisingly, animals attempted to open their pneumostome to breathe-air more often the longer the duration of the training session. What might have been surprising is that even though the snails that were subjected to the 0.25 -hr duration training sessions received far fewer contingent reinforcing stimuli, they were still able to form the association (i.e. learn) and exhibit both LTM and ITM. The smaller number of reinforcing stimuli presented to the 0.5- or 0.25 -hr group once a day for 5 days may be one explanation for their inability to learn. However, the fewer number of breathing attempts did not hinder the ability to learn or remember when two or more training sessions (separated by either a 0.5 - or a 1-hr interval) were delivered per day. The fewer number of attempted pneumostome openings and contingent reinforcing stimuli in the $0.25-\mathrm{hr}$ groups as opposed to the $0.5-\mathrm{hr}$ groups did not appear to alter the formation of either ITM or LTM. This observation is one reason why we chose to present normalized data. If either ITM or LTM were different because of the reduced training time this difference would have been easily seen. However, we saw no such difference. We can only conclude that a $0.25-\mathrm{hr}$ training period is sufficient to produce learning and that, depending on other factors (e.g., interval between training sessions), either ITM or LTM will be produced. To date, we have not attempted to use shorter training sessions to see whether, for example, a 10- min training session is sufficient to produce learning and ITM or LTM. It is possible that such a short period will not be adequate for the production of learning and memory. We have also not attempted experiments in which we increase the interval between training sessions to 5 or $6 \mathrm{hrs}$, for example, to determine whether learning and memory occur or whether they are improved.

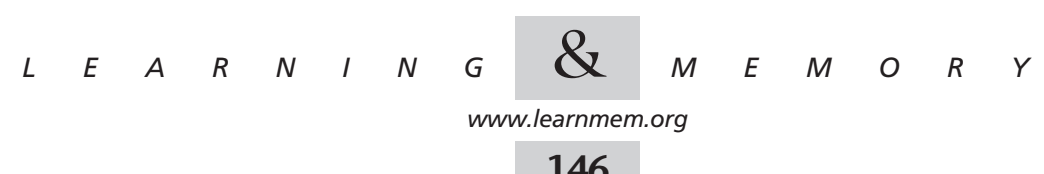


There did not appear to be any obvious or consistent change in the time interval between air-breathing attempts. Some snails attempted a number of breaths in rapid sequence, whereas others did not, and the sequence varied across the training sessions for individual snails. Thus, we cannot come to any conclusion regarding the intervals between air-breathing attempts and the acquisition of learning and memory. In a series of preliminary experiments, we found, however, that the animals do not appear to be able to form an association (i.e., learn) if the reinforcing stimulus is delivered on alternative pneumostome openings. Further experimentation is necessary to determine whether such a different schedule of reinforcement significantly affects the ability to learn and form memory. Another obvious variable that could potentially affect the establishment of learning and its memory is the strength of the tactile stimulus delivered to the pneumostome by the experimenter. We have made every attempt to be consistent in how much force we use and have picked a force that causes a rapid closure of the pneumostome. Previously (Lukowiak et al. 1996), we used an explicitly strong tactile stimulus as a control for this variable and saw no obvious alteration in the snail's learning or memory. We also made use of a similar explicitly strong tactile stimulus in the yoked control preparations and saw no learning in these animals. We have, however, performed pilot experiments in which we employed a very weak tactile stimulus to the pneumostome. This weak stimulus caused a slow or incomplete closing of the pneumostome. In these preliminary experiments, the animals attempted to open their pneumostome far more often and did appear to learn. More complete experimentation addressing this point is necessary.

We tested animals to determine the number of training sessions required for animals to learn and exhibit LTM when shorter duration training sessions are used. Little information has been gathered previously on this subject. Shorter training session durations are sufficient to elicit both learning and LTM, but the training sessions have to occur more frequently than once a day. In fact, the learning curves produced with the shorter duration training periods were remarkably similar to the learning curve produce in the initial report on operant conditioning of this behavior (Lukowiak et al. 1996). Obviously, the interval between training sessions plays some important and not yet understood role in the production of the formation of the association (i.e., learning) and its retention (i.e., the consolidation process). We will begin to examine this in semi-intact preparations where we can monitor the behavior and neural activity of the central pattern generator (CPG) neurons simultaneously and determine the changes occurring in the neurons as the preparation begins to learn and remember.

We also showed that both learning and LTM could result from a single days of training. Three sessions on one day (with each of the three duration training sessions) were sufficient to result in learning and LTM. However, for LTM to result when either the 0.25 - or 0.5 -hr training sessions were used, the SI had to be at least $1 \mathrm{hr}$. An SI of $0.5 \mathrm{hr}$ was inadequate to produce LTM, although it was sufficient to enable learning to occur. That these training procedures lead to a form of associative learning was demonstrated by employing the yoked control procedure. When these animals received the tactile stimulus to the pneumostome area that was not contingent on the opening of its pneumostome, no learning occurred. Again, these data are consistent with our earlier published data showing that this is indeed a form of associative learning (Lukowiak et al. 1996, 1998; Spencer et al. 1999).

A number of points can be drawn from the data discussed above. (1) The total duration of training necessary to produce learning and LTM on a single day can be as little as $0.75 \mathrm{hr}$ if there is an adequate interval between training sessions. (2) The learning curves produced by the various procedures are virtually indistinguishable from one another. (3) The absolute number of breathing attempts that occur does not appear to alter the ability of the snails to learn and remember, except for sessions occurring once a day. (4) Operant conditioning (i.e., associative learning) does not necessarily lead to LTM, thus demonstrating that learning and memory are not the same process. (5) Presentation of the tactile stimulus to the pneumostome does not itself result in learning and memory. There has to be a specific contingency (i.e., yoked controls do not show learning and memory).

Was there any retention of the learned behavior when the 0.5 -hr duration SI was used with either the 0.25- or $0.5-\mathrm{hr}$ duration training sessions? Yes, but it only persisted for at most 2-3 hr. This new finding for Lymnaea preparations fits in well with the scheme put forward by Rosenzweig et al. (1993) regarding the existence of ITM. This memory is a shorter form of memory than LTM, lasting only a few hours; but it appears to share certain of the same characteristics of LTM. The most important of these is its requirement for new protein synthesis. Whether the processes underlying ITM and LTM are serial or parallel remains to be conclusively determined. Biochemical evidence suggests that they are parallel but related processes (Mauelshagen 1998; see below). Typically ITM persists for only a few hours. It may serve a function somewhat analogous to a memory cache of a computer. ITM may allow a memory to be maintained until such time as LTM can be induced and stored. After such a period, ITM disappears (the mechanism of this event is unknown), as it is no longer necessary. If ITM were not present, the establishment of LTM might possibly be more difficult. However, this attractive hypothesis needs testing.

Although this study is the first time to our knowledge that ITM has been shown to exist in Lymnaea, it is not the first time it has been demonstrated in invertebrates. Classi-

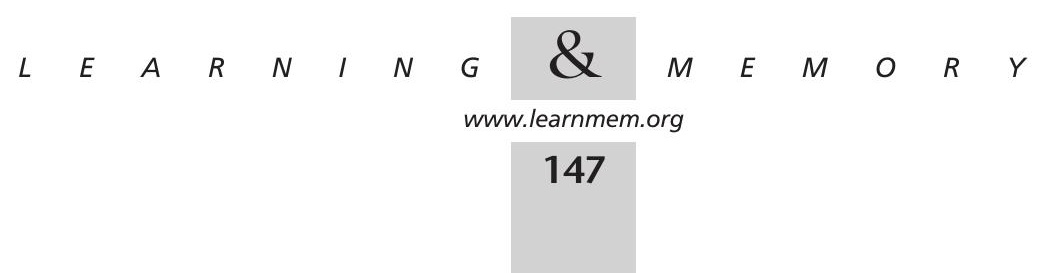


cal conditioning of honeybees has shown similar effects of altering the time interval between learning trials (Gerber et. al. 1998). In Aplysia, Susswein and colleagues have also demonstrated a form of ITM in food aversion studies (Botzer et al. 1998). The neural correlates and/or neural causes of ITM are not yet known. However, it is from the study of neural analogs of memory that we have gained most of our knowledge of the possible mechanism(s) of ITM.

One of the first demonstrations of an ITM-like phenomenon at the neural level was that of Ghirardi et al. (1995). In that study the authors showed that an intermediate form of facilitation could be produced at the Aplysia sensory motor neuron synapse in culture even though transcriptional blockers were present. That is, this intermediate form of synaptic plasticity, although dependent on new protein synthesis, was not dependent on the process of transcription. More recent studies in Hermissenda have also shown that there exists an intermediate phase of memory dependent on protein synthesis but not mRNA synthesis (Crow et. al. 1999). The switch from STM (lasting only minutes) to LTM is hypothesized to involve the cAMP/calcium response element (CRE)-binding protein (CREB) leading to altered gene expression in neurons. CREB, being a regulator of mRNA synthesis, does not likely play a role in ITM. It seems likely, then, that the new proteins necessary for ITM are produced from pre-existing mRNA transcripts. Complementary data have also been obtained in three other studies (Martin 1997; Manseau 1998; Mauelshagen 1998). These data suggest further that message, which may already be present in extrasomal areas (i.e., the unpolarized axon in invertebrates), when translated, serves to mediate the intermediate form of synaptic plasticity. This is certainly a fast and economical method of altering synaptic plasticity of intrinsic membrane properties of neurons. Isolated Lymnaea axons (i.e., without the soma) have the ability to synthesize de novo integral functional membrane proteins (Van Minnen 1997; Spencer et al. 1999b).

In a study by Mauelshagen et al. (1998), the absence of a neural analog of LTM formation after massed training did not affect the appearance of a neural analog of ITM. This result suggests that these two memory processes are not sequentially dependent. Previous research has shown that the formation of ITM precedes the formation of LTM. If ITM is dependent on already existing mRNA that will be translated at the site of the specific synapse undergoing change, for example, then it should occur faster than LTM, which is dependent on nuclear events and transport of the protein(s) to the site of plasticity. It has also been shown that these two memories depend on different neurochemical processes, specifically, on different classes of protein kinase activities (Rosenzweig et al. 1993). Different protein kinases apparently play different roles in the mediation of different aspects of synaptic plasticity at the Aplysia sensory motor neuron synapse in culture (Manseau et al. 1998). Whether or not these different pathways subserve LTM and ITM is not clear.

An intriguing question concerns why there must be sufficient time between training sessions to allow the consolidation process. Several hypotheses have been proposed. One plausible explanation involves many different isoforms of CREB (Carew 1996). CREB exists in several isoforms, some of which are activators and others that are repressors of gene activity. It is postulated that the repressor isoforms of CREB become inactive much more quickly than do the activator forms during the rest period between training events. This difference results in a net surplus of CREB activators, which leads to the induction of LTM. During massed trials, however, the rapidly occurring next trial causes a reactivation of the CREB repressors. The repressor isoform now blocks the induction of LTM. However, this explanation may not be sufficient to explain why a 1-hr duration training session once a day is sufficient for both learning and LTM. Whether such a scheme will ultimately explain the occlusion of LTM by massed training procedures remains to be determined.

In conclusion, this study has established effective training procedures that allow us to examine both ITM and LTM. Significant effects on the establishment of learning and memory are found when changes are made to either the number of training sessions, the duration of each session, or the amount of time given between each session. LTM was only produced when the interval between the training sessions was $1 \mathrm{hr}$. A shorter interval between the training sessions instead led to the establishment of ITM. Thus we have now established a model that will allow for the analysis of the neural mechanism(s) responsible for this form of memory.

\section{MATERIALS AND METHODS}

Lymnaea stagnalis originally obtained from Vrije University in Amsterdam were laboratory bred in our snail facility. In the studies reported here we used snails with shell sizes of $25-30 \mathrm{~mm}$. The operant conditioning procedure initially used was similar to that used previously (Lukowiak 1996). Briefly, snails were individually marked to allow experimenters to identify them. To increase aerial respiratory behavior animals were placed into $500 \mathrm{ml}$ of hypoxic pond water in a $1000-\mathrm{ml}$ glass beaker. The pond water was made hypoxic by bubbling $\mathrm{N}_{2}$ through it for $20 \mathrm{~min}$ prior to placing the animals in the beaker. In all experimental and yoked control procedures, animals were initially allowed a 10-min acclimation period in the hypoxic water during which time they were free to perform aerial respiration ad libitum. At the end of this period, each animal was gently pushed down beneath the water surface, ensuring all snails were under water before the training/testing began.

During the training period, when a snail reached the surface of the water and opened its pneumostome, a tactile stimulus (delivered with a hand-held sharpened wooden applicator, our digital stimulator) was presented to the pneumostome area each time the animal attempted to open its pneumostome. Stimulation of the pneumostome area evoked the whole-animal withdrawal response (Inoue et al. 1996), which caused the pneumostome to immediately close. The time of each tactile stimulus to the pneumostome

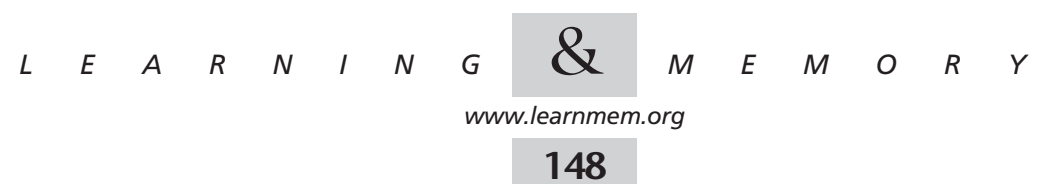



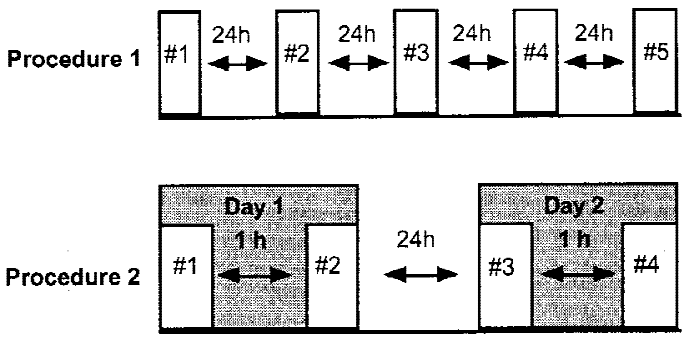

$0.25 \mathrm{~h}$

$0.5 \mathrm{~h}$

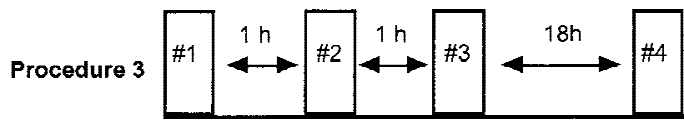

$0.25 h$

$1,0 \mathrm{~h}$

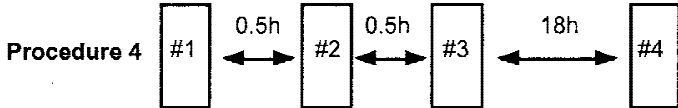

$0.25 h$

$1.0 \mathrm{~h}$

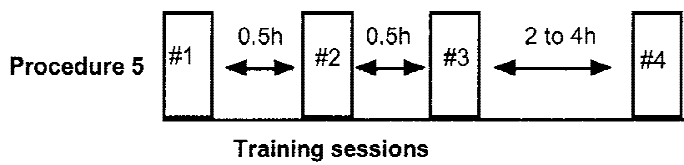

$0.25 h$

$0.5 \mathrm{~h}$

Figure 10 Schematic diagram illustrating the five different training protocols used. In each procedure, the training session duration is given. Training session durations were $0.25,0.5$, or $1.0 \mathrm{hr}$. The interval between each training session (SI) is also illustrated, as is the interval between the final training session and the test session.

was recorded for each animal. At the end of each session we calculated the number of times each animal attempted to open its pneumostome and summed all the animals together to calculate the total number of openings attempted by the cohort of animals for that particular session.

Directly after the training or yoked control period (see below) animals were returned to a beaker of normoxic water.

\section{Tests to Determine Memory}

We operationally defined a cohort of animals as exhibiting LTM when the number of attempted pneumostome openings in the session $18 \mathrm{hr}$ after the last training session were not significantly different from each other, but were significantly different from the number of attempted openings in session 1. In a similar manner, we operationally defined ITM in the same manner only in this case the time interval was 2,3 , or $4 \mathrm{hr}$. Thus, for example, ITM would be shown to occur if the number of attempted openings on trial 4 ( 2 hr after trial 3) was not significantly different from the response in session 3 but both of these responses were significantly different from that in session 1 . We chose these definitions because all animals were naive in session 1 and only began to form an association with contingent reinforcement. The test session following training was always of the same duration as the training session in each procedure.

\section{Training Procedures}

Five different training protocols were used (see Fig.10), differing in
(1) the frequency of training sessions per day(2) the duration of the training session and (3) the time interval (between each training session(SI). During the SI, animals were placed in normoxic pond water.

\section{Procedure 1}

Three cohorts of animals were trained once a day for 5 consecutive days. In the first cohort, the duration of the training session was $0.25 \mathrm{hr}$. The second cohort had a training session duration of $0.50 \mathrm{hr}$. Finally, the third cohort of animals had a training session duration of 1.0

\section{Procedure 2}

Two cohorts of animals were used. Animals were trained twice a day for two days, with an SI of $1 \mathrm{hr}$. One cohort's training session duration was $0.25 \mathrm{hr}$ while the other cohort's training session was $0.5 \mathrm{hr}$.

\section{Procedure 3}

Three cohorts of animals were trained with three sessions on a single day. They were then tested for LTM on the following day ( $>18 \mathrm{hr}$ ). The SI for each cohort was $1 \mathrm{hr}$. The first cohort had a training session of $0.25 \mathrm{hr}$ duration the second cohort had a training session of $0.5 \mathrm{-hr}$ and the third cohort had a training session duration of $1 \mathrm{hr}$.

\section{Procedure 4}

This procedure was similar to procedure 3 except that the SI was 30 min rather than $1 \mathrm{hr}$. Again, there were three cohorts of animals receiving $0.25-, 0.5-$, or 1-hr training sessions respectively.

\section{Procedure 5}

This procedure was similar to the massed training procedure (an SI of $0.5 \mathrm{hr}$ ). However, in this procedure only 0.5 - and 0.25 -hr duration training sessions were used. Separate groups of animals from each 0.5 - or 0.25 -hr cohort were tested for the retention of memory 2 or $3 \mathrm{hr}$ after the third and final training session. A separate group of animals from the $0.25-\mathrm{hr}$ cohort were also tested $4 \mathrm{hr}$ after the last training session. This procedure tested for ITM.

\section{Yoked Control Procedures}

To show that the changed behavior subsequent to the training procedure arose as a consequence of operant conditioning we used a yoked control procedure for complete details (see Lukowiak et al. 1996. Briefly, two different yoked control procedures were used. In the first series of control experiments where a 1-hr training interval was used (e.g., procedure 3 ) naïve animals received a poke to the pneumostome area whenever the animal to which they were yoked attempted to open its pneumostome. Then, we then tested the response of these animals $18 \mathrm{hr}$ later. In this test period, the animals received a poke to the pneumostome every time they attempted to open their pneumostome. We then compared the number of pokes received in this session to the number of pokes received by the operantly conditioned animals in session 1 . In the second series of yoked controls (using the 0.5-and 0.25-hr training sessions) we initially determined the number of pokes received by the control group. In this pre-test the control animals were poked every time they attempted to open their pneumostome. Then we allowed a 24-hr period to elapse before poking their pneumostome whenever

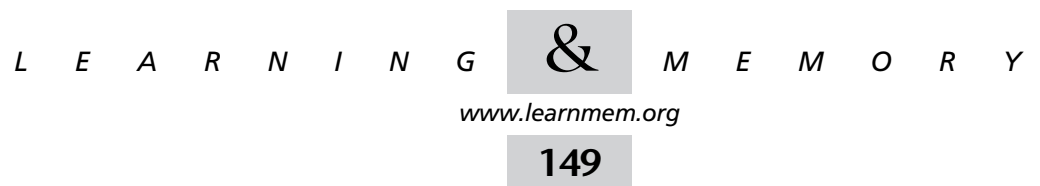


the animal to which they were yoked received a poke (using procedure 3). Then we tested the control animals $18 \mathrm{hr}$ later. In this session they received a poke every time they attempted to open their pneumostome to breathe (the post-test). We compared the number of pokes they received in this post-test session with the number they received in the pre-test session.

\section{Data Analysis and Statistics}

For the purposes of graphing the data, we normalized the data with $100 \%$ being the initial number of pokes to the pneumostome area for each animal in session 1 . The mean \pm the standard error of the mean (S.E.M.) were plotted for each session. The actual number of pokes and thus the number of air-breathing attempts, were recorded for each cohort of animals tested. As one might expect, the longer the training period, the greater the number of stimuli delivered.

Statistical analysis consisted of an ANOVA for each cohort followed by a post hoc Fisher's LSD (protected $t$-test) for each session being compared. Differences were deemed to be significant when $P<0.05$.

\section{ACKNOWLEDGMENTS}

N.A. and D.K. are supported by Alberta Heritage Foundation for Medical Research (AHFMR) summer studentship awards. NS is a AHFMR Scientist. This work was supported by a grant from Faculty of Medicine Endowment Fund (Ruth Rannie Memorial Endowment Fund).

The publication costs of this article were defrayed in part by payment of page charges. This article must therefore be hereby marked "advertisement" in accordance with 18 USC section 1734 solely to indicate this fact.

\section{REFERENCES}

Abel, T., K. Martin, D. Bartsch, and E. Kandel. 1998. Memory suppressor genes: Inhibitory constraints on the storage of long-term memory. Science 279: 338-341.

Botzer, D., S. Markovich, and A. Susswein. 1998. Multiple memory processes following training that a food is inedible in Aplysia, Learning and memory 5: 204-219.

Carew, T.J. 1996. Molecular enhancement of memory formation. Neuron 16: 5-8.

Carew, T., H. Pinsker, and E. Kandel. 1972. Long term habituation of a defensive withdrawal response reflex in Aplysia. Science 175: 451-454

Crow, T., J.-J. Xue-Bain, and V. Siddiqi. 1999. Protein synthesis-dependant and mRNA synthesis-independent Intermediate phase of memory in Hermissenda. J. Neurophysiol. 82: 495-500.

Dickinson, A. 1980. Contemporary animal learning. Cambridge University Press, Cambridge, UK.

Gerber, B., D. Wustenberg, A. Schutz, and R. Menzel. 1998. Temporal determinants of olfactory long-term retention in honeybee classical conditioning: Non monotonous effects of the training trial interval. Neurobiol. Learning Mem. 69: 71-78.

Ghirardi,M., P. Monterolo, and E. Kandel. 1995. A novel intermediate stage in the transition between short- and long-term facilitation in the sensory to motor neuron synapse of Aplysia. Neuron 14: 413-420.

Hermitte, G., M.E. Pedreira, D. Tomsic, and H. Maldonada. 1999. Context shift and protein synthesis inhibition disrupt long-term habituation after spaced, but not massed, training in the crab Chasmagnathus. Neurobiol. Learning Memory 71: 34-49.

Inoue, T., M. Takasaki, K. Lukowiak, and N. Syed. 1996. Inhibition of the respiratory pattern-generating neurons by an identified whole-body withdrawal interneuron of Lymnaea stagnalis. J. Exp. Biol. 199: $1887-1898$

Lechner, H.A., L.R. Squire, and J.H. Byrne. 1999. One-hundred years of consolidation-remembering Muller and Pilzecker. Learning $\varepsilon$ Memory 6: 77-87.

Lukowiak, K. E. Ringseis, G. Spencer, W. Wildering, and N. Syed. 1996 Operant conditioning of aerial respiratory behaviour in Lymnaea stagnalis. J. Exp. Biol. 199: 683-691.

Lukowiak, K., R. Cotter, J. Westly, E. Ringseis, G. Spencer, and N. Syed. 1998. Long-term memory of an operantly conditioned respiratory behaviour pattern in Lymnaea stagnalis. J. Exp. Biol. 201: 877-882.

Lukowiak, K., N. Syed, and G. Spencer. 1999. Operant conditioning of aerial respiratory behaviour in Lymnaea-Results from semi-intact preparations. Soc. Neurosci. Abstracts 25: 530.8

Mackintosh, N.J. 1974. The psychology of animal learning. Academic Press, New York, NY.

Manseau, F., W. Sossin, and V. Castellucci. 1998. Long-term changes in excitability induced by protein kinase C activation in Aplysia sensory neurons. J. Neurophysiol. 79: 1210-1218.

Martin, K., A. Casadio, H. Zhu, E. Yaping, J. Rose, M. Chen, C. Bailey, and E. Kandel. 1997. Synapse specific, long-term facilitation of Aplysia sensory to motor synapses: A function for local protein synthesis in memory storage. Cell 91: 927-938.

Mauelshagen, J., C.M. Sherff, and T.J. Carew. 1998. Differential induction of long-term synaptic facilitation by spaced and massed applications of serotonin at sensory neuron synapses of Aplysia californica. Learning E Memory 5: 246-256.

Milner, B., L. Squire, and E. Kandel. 1998. Cognitive neuroscience and the study of memory. Neuron 20: 445-468.

Mpitsos, G.J. and K. Lukowiak. 1985. Associative and nonassociative learning in gastropod molluscs. In The Mollusca, Neurobiology and behavior (ed., A.O.D. Willows) pp. 95-267. Academic Press, New York, N.Y.

Nargeot, R., D. Baxter, and J. Byrne. 1999. In vitro analog of operant conditioning in Aplysia. I. Contingent reinforcement modifies the functional dynamics of an identified neuron. J. Neurosci. 19: $2247-2260$.

Rosenzweig, M.R., E.L. Bennett, P.J. Colombo, D.W. Lee, and P.A. Serrano. 1993. Short-term intermediate term and long-term memories. Behaviour Brain Res. 57: 193-198.

Rowe, M.K. and M.G. Craske. 1998. Effects of expanding-spaced vs massed exposure schedule on fear reduction and return of fear. Behaviour Res. Therapy 36: 701-717.

Syed, N., A. Bulloch, and K. Lukowiak. 1990. In vitro reconstruction of the respiratory central pattern generator (CPG) of the mollusc Lymnaea. Science 250: 282-285.

Syed, N., R. Ridgway, K. Lukowiak, and A. Bulloch. 1992. Transplantation and functional integration of an identified respiratory interneuron in Lymnaea stagnalis. Neuron 8: 767-774.

Spencer, G.E., N. Syed, and K. Lukowiak. 1999a. Neural changes after Operant conditioning of the aerial respiratory behavior in Lymnaea stagnalis. J. Neurosci. 19: 1836-1843.

Spencer, G., N. Syed, E. van Kesteren, K. Lukowiak, W. Geraerts, W., and J. van Minnen. 1999b. Expression and functional integration of a neurotransmitter receptor in isolated axons of identified Lymnaea neurons. Soc. Neurosci. Abstr. 25: 175.19.

Tully, T. 1998. Toward a molecular biology of memory: The light's coming on Nature Neurosci. 1: 543-545.

Van Minnen, J., E. Bergman, R. Van Kesteren, A. Smit, W. Geraerts, K. Lukowiak, S. Hasan, and N. Syed. 1997. De novo protein synthesis in isolated axons. Neuroscience 80: 1-7.

Received November 3 1999; accepted in revised form April 5, 2000.

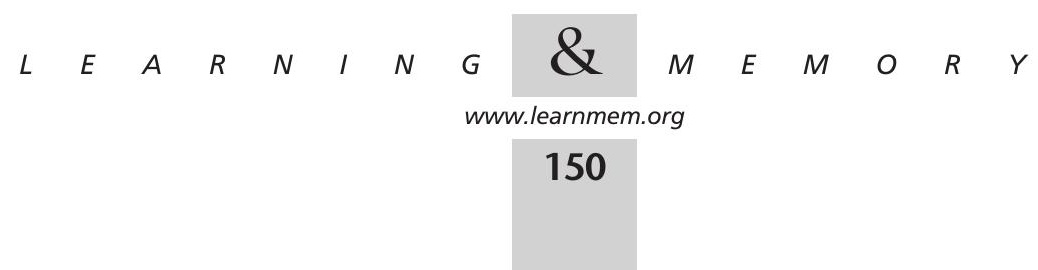




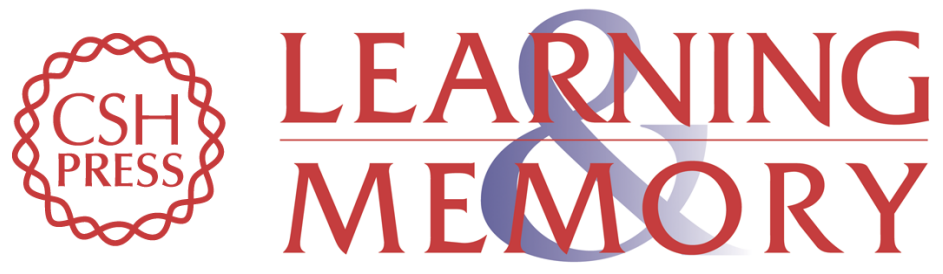

\section{Operant Conditioning in Lymnaea: Evidence for Intermediate- and Long-term Memory}

Ken Lukowiak, Nimet Adatia, Darin Krygier, et al.

Learn. Mem. 2000, 7:

Access the most recent version at doi:10.1101//m.7.3.140

References This article cites 25 articles, 11 of which can be accessed free at: http://learnmem.cshlp.org/content/7/3/140.full.htmI\#ref-list-1

License

Email Alerting Receive free email alerts when new articles cite this article - sign up in the box at the Service top right corner of the article or click here. 